\title{
Investigation of Relation between Fracture Scale and Acoustic Emission Time-Frequency Parameters in Rocks
}

\author{
Junwen Zhang $\mathbb{1}$ \\ College of Resource and Safety Engineering, China University of Mining and Technology (Beijing), Beijing 100083, China \\ Correspondence should be addressed to Junwen Zhang; zhangjunwen1977@163.com
}

Received 27 October 2017; Accepted 31 January 2018; Published 19 March 2018

Academic Editor: Longjun Dong

Copyright ( 2018 Junwen Zhang. This is an open access article distributed under the Creative Commons Attribution License, which permits unrestricted use, distribution, and reproduction in any medium, provided the original work is properly cited.

\begin{abstract}
To investigate relation between fracture scale and acoustic emission time-frequency parameters in rocks, experiments of acoustic emission monitoring of granite uniaxial compression were carried out. The AE signal energy and dominant-frequency of granite fracture process were extracted by means of AE time-frequency analysis. The relation between fracture scale and AE time-frequency parameters (energy and frequency) in granite fracture process was analyzed. The evaluation model of rock fracture scale based on $\mathrm{AE}$ energy and dominant-frequency was established by using the intrinsic relation between the scale of rock fracture and the timefrequency parameters of rock mass. The evolution of crack scale in the process of uniaxial compression was analyzed based on the evaluation model of rock fracture scale. Results show that the AE energy and the dominant-frequency can reflect the crack scale inside the rock. The scale of rock fracture is proportional to the AE energy, which is inversely proportional to the AE dominantfrequency. Signals with low frequency and high energy usually represent large-scale cracks. On the contrary, if the high frequency has low energy value, it indicates a small-scale crack. The theory and method of evaluation of rock rupture scale based on AE timefrequency information (energy, frequency) can describe the failure process of rock crack scale variation characteristics. It provides a way and method for investigating the characterization of fracture size evolution process of rock fracture.
\end{abstract}

\section{Introduction}

The phenomenon that the rock releases stress-strain energy in the form of elastic waves during the process of stress catastrophe is called acoustic emission (AE). The rock failure is actually the process that the initial fissures are closed and the cracks are gradually generated, expanded, and penetrated. The acoustic emission/microseismic monitoring technologies can monitor rock fracture process in real time and have been widely used in the study of rock failure process and instability information $[1,2]$. As an attendant phenomenon in the process of rock failure, rock acoustic emission contains many information of rock internal fracture process [3]. Therefore, studying the relation between acoustic emission parameters and rock fracture during the rock failure process and establishing the relation model between acoustic emission information and rock fracture characteristics (model, scale, etc.) are helpful in understanding the rock failure mechanism and have important scientific research significance and engineering significance to deeply comprehend the rock dynamic disaster mechanism.

There are two analysis methods of acoustic emission information of rock fracture process. (1) Parametric analysis: the parameter analysis method is based on the recorded time-domain waveform of acoustic emission signal; then several related statistical data are directly extracted by the system to simplify the waveform characteristic parameters to represent the characteristics of acoustic emission signal, so the relevant information on acoustic emission sources can be analyzed and processed. Commonly used acoustic emission parameters include the number of events, ring count, absolute energy, amplitude, duration, rise time, and threshold. Parameter analysis is currently the most commonly used method for analyzing acoustic emission signal. Many of the characteristics of acoustic emission sources can be described with these parameters, which are easy and quick in practical application. 
The parametric analysis of acoustic emission was used in [4-6]. Cai et al. [7] analyzed rock acoustic emission characteristics in laboratory test to get the initial crack initiation time and the relation between rock failure process and stress according to the acoustic emission event. Yu et al. [8] conducted direct tensile, splitting, and uniaxial compression tests and analyzed the acoustic emission characteristics of rock samples under several stress states. Li et al. [9] performed uniaxial compression experiment to study the relation between the number of acoustic emission events (AE number), event rate and stress, and time. Yang et al. [10] carried out triaxial compression acoustic emission tests on limestone, by using acoustic emission parameters (acoustic emission ringing count and acoustic emission energy); the damage evolution characteristics were analyzed. The above results lay the foundation for the study of rock acoustic emission. However, the acoustic emission parametric analysis only simply described the waveform characteristics and provided limited information of acoustic emission source, which can not accurately and comprehensively reflect the characteristics of the acoustic emission signal. Therefore, the time-domain statistical parameters of the acoustic emission signal of rock fracture process are of great randomness, and the parameters characteristics of rock with different lithology are similar, which makes it difficult to predict rock fracture. Therefore, studying the mechanism of rock failure process from the angle of acoustic emission parameters analysis is not very satisfactory for the prediction of rock burst so far [11]. (2) Waveform analysis: waveform analysis is based on acoustic emission time-domain waveform and signal spectrum analysis method to obtain the waveform spectrum information of acoustic emission signal. The acoustic emission waveform carries all information such as the stress state, structure, and physical and mechanical properties of the rock. The analysis of the waveform information can better understand the rock failure mechanism and precursor [12]. In recent years, scholars began to study the rock failure mechanism from the perspective of acoustic emission waveform analysis, which aims to find the necessary and sufficient conditions for rock failure. Ji et al. $[13,14]$ found that the dominantfrequency scale of granite acoustic emission signal is 41 $85 \mathrm{kHz}$, and as the amplitude of the acoustic emission signal increased, so does the stress level. Miao et al. [15] studied the acoustic emission spectral characteristics of limestone and marble indoor rock burst simulation experiments and pointed out that the initial loading showed high frequency and low amplitude, and rock burst showed low frequency and high-amplitude. This demonstrates that a large number of high-amplitude acoustic emission signals are generated when the rock is approaching the main fracture [16]. The most prominent research result in the acoustic emission spectrum analysis of rock mass is that the rock AE dominantfrequency moves from high frequency to low frequency with the stress level increasing [17-19]. Dong et al. [20, 21] proposed acoustic emission source location methods without the need of premeasured wave velocity to improve the source location accuracy. The characteristics of acoustic emission waveform are directly affected by the type of acoustic emission sources, and acoustic emission signals demonstrate different characteristics when the scale of cracking, scale, and type are varied. Therefore, the study of the spectrum characteristics of rock acoustic emission signal should focus on the corresponding relation between the properties (scale, type) of crack expansion of rock fracture process and the characteristics of acoustic emission signal (energy, amplitude, and dominant-frequency) and then understand the physical mechanism generated by the different modes of acoustic emission signals, which is of great significance to reveal the nature of rock fracture process.

Focusing on the relation between rock fracture scale and acoustic emission information, scholars have conducted some relevant researches. The $b$-value of the acoustic emission of rock fracture process can characterize the fracture scale; when the $b$-value is big, the small-scale microfracture dominates, while when the $b$-value is small, the large-scale fracture occupies more proportion [22]. The theoretical study of seismology suggests that there is a certain degree linear relation between the source scale and the frequency [23, 24]. The larger the source scale is, the lower the frequency of the generated signal. The existing studies have shown that the spectrum characteristics of acoustic emission are related to the inherent properties of rock $[25,26]$. The rock fracture scale has natural correspondence with frequency, but currently there is not a unified understanding of the relation between rock fracture scale and the acoustic emission frequency-domain characteristics. At the same time, the researches of scholars often focused on the relation between the single acoustic emission parameters and the rock fracture scale, and discussed the correspondence. The relation between rock fracture scale and multiple acoustic emission information (time-frequency) characteristics has not yet been under systematic and in-depth study, neither a classification method of rock fracture scale based on acoustic emission time-frequency information is proposed.

Based on this, the experiment of acoustic emission monitoring of granite uniaxial compression was carried out. The AE signal time-domain and frequency-domain information of granite fracture process are extracted by means of parametric analysis and waveform analysis. The variation features of the acoustic emission energy and the dominant-frequency of the granite fracture process as well as the relation between fracture scale and two AE time-frequency parameters (energy and frequency) are analyzed. The evaluation model of rock fracture scale based on AE energy and dominant-frequency is established by using the correspondence relation between the scale of rock fracture and the AE time-frequency information. On the basis of this, the evolution of crack scale in the process of rock uniaxial compression is analyzed based on the evaluation model of rock fracture scale. Finally, the theory and method of acoustic emission evaluation of rock fracture scale are proposed, which provide the theoretical basis for quantitatively characterizing the crack scale of rock fracture process.

\section{Experimental Design}

2.1. Experimental Samples and Equipment. The experimental granite is from Laizhou, Shandong, made into $50 \times 50 \times$ 


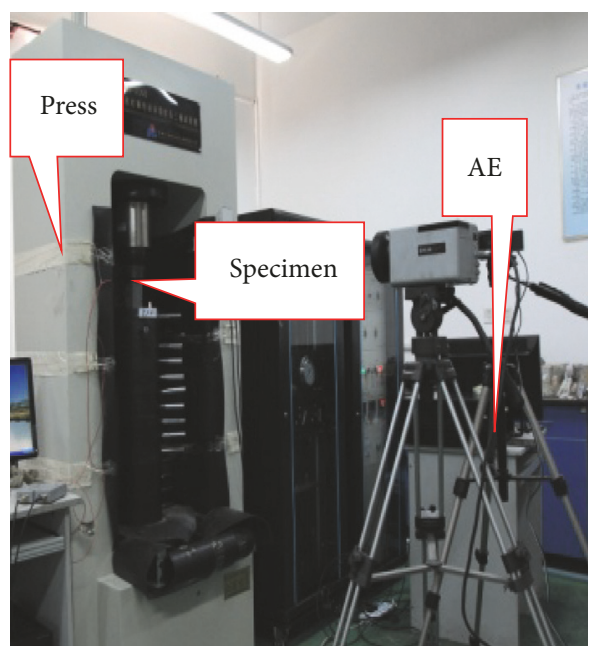

(a) Experimental site

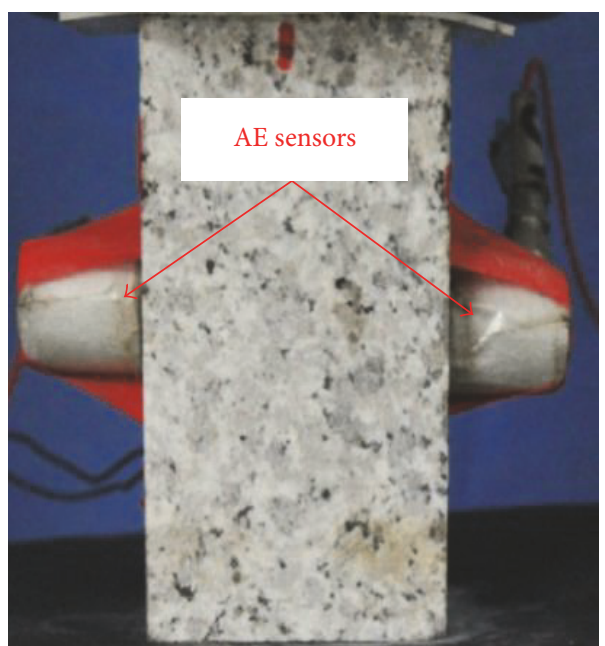

(b) AE sensors layout plan

FIGURE 1: Experimental system.

$100 \mathrm{~mm}$ standard rectangular sample. The unevenness error of two sides of the rock specimen is less than $0.05 \mathrm{~mm}$, and the length error of two sides is less than $0.3 \mathrm{~mm}$. Considering that the rock mass of engineering practice is always hosted in a certain groundwater environment and is often in a saturated state, the granite is subjected to saturated water treatment. According to the Water and Hydropower Engineering Rock Test Rules, the saturated water treatment is conducted by free water absorption method. The detailed process is as follows: put the sample into the tank, dump water to the $1 / 4$ height of sample, and then to the $1 / 2$ and $3 / 4$ height of sample every $2 \mathrm{~h}$, submerge all samples after $6 \mathrm{~h}$, and finally place the sample in the water for $48 \mathrm{~h}$. The percentage of saturated water content of granite is $0.149 \% \sim 0.167 \%$. Five samples were selected to carry out the experiment. The samples numbers were HSHG1, HSHG-2, ..., HSHG-5.

The loading system used in the experiment is the TAW3000 servo rock mechanics test system. The acoustic emission is the PCI-2 multichannel acoustic emission monitoring system produced by PAC in the United States. Two acoustic emission sensors are placed in the experiment. Experimental site is shown in Figure 1(a); sensors layout is shown in Figure 1(b).

2.2. Experimental Process. In this experiment, the axial displacement control method is used to load. To ensure that the specimen is in complete contact with the loading surface and avoid the contact noise affecting the acoustic emission monitoring results, firstly, preload to $1.5 \mathrm{kN}$ and then load at the rate of $0.2 \mathrm{~mm} / \mathrm{min}$ until damage. The acoustic emission system is used to synchronously monitor the fracture process of the sample in real time. In order to eliminate the impact of the external environment, the press and the acoustic emission host are grounded to eliminate the noise caused by current. The experimental preamplifier threshold (gain) is $40 \mathrm{~dB}$ and the acoustic emission sampling threshold is $45 \mathrm{~dB}$. People are prohibited to move and talk after the start of the experiment.
The sampling frequency of the acoustic emission instrument is set to $1 \mathrm{kHz} \sim 3 \mathrm{MHz}$, the sampling rate of acoustic emission waveform is $1 \mathrm{MSPS}$, the pretrigger is 256 , and the length is $2 \mathrm{~K}$. The acoustic emission sensor is $\mathrm{R} 6 \alpha$ type resonant high sensitivity sensor; the frequency response range is 0 $300 \mathrm{kHz}$. During the experiment, Vaseline is applied between the sensor and the coal sample to enhance their coupling and reduce the attenuation of the acoustic emission signal.

\section{The Method of Time-Frequency Information Extraction of AE Signal}

The analysis of acoustic emission characteristic parameters is performed to find the characteristic and intrinsic law of acoustic emission source, which is one of the most basic methods to analyze the damage of material or structure. Among them, the acoustic emission waveform characteristics are generally considered to be an effective way to reflect the fracture failure scale. The characteristic parameters of acoustic emission signal include ringing count, amplitude, energy, rise time, and dominant-frequency, as shown in Figure 2.

3.1. Time-Domain Information Extraction. Based on the parametric analysis method, the acoustic emission event rate and the acoustic emission energy rate are selected to characterize the time-domain waveform of the acoustic emission signal.

(1) Acoustic Emission Event Rate. The acoustic emission events are signals whose amplitude exceed the threshold and allow the monitoring channel to acquire data. The number of signals detected at a certain moment is the number of acoustic emission events. The acoustic emission event rate is the number of acoustic emission events per unit time. It can quantitatively describe the law of crack propagation in the 


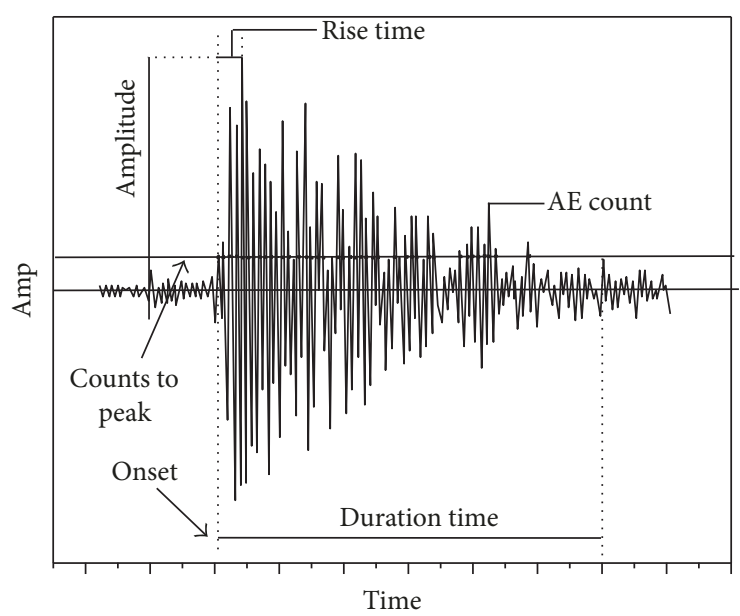

FIgURe 2: Acoustic emission signal.

rock: the high acoustic emission event rate indicates that there are more cracks in the rock sample.

\section{(2) Acoustic Emission Absolute Energy Rate}

Absolute Energy. It is a true reflection of the energy of the acoustic emission impact signal. It reflects the energy released by a cracked source in the form of elastic waves in an acoustic emission event. The unit of energy is attojoules (abbreviated as aJ); 1 aJ corresponds to $10^{-18} \mathrm{~J}$. According to the definition of signal energy, the area enclosed by the squared amplitude of acoustic emission signal can be used as a measure, and the energy of acoustic emission signal can be calculated by root mean square voltage $\left(V_{\mathrm{rms}}\right)$ or mean square voltage $\left(V_{\mathrm{ms}}\right)$ :

$$
\begin{aligned}
V_{\mathrm{ms}} & =\frac{1}{\Delta T} \int_{0}^{\Delta T} V^{2}(t) d t, \\
V_{\mathrm{rms}} & =\sqrt{V_{\mathrm{ms}}}
\end{aligned}
$$

where $\Delta T$ is average time; $V(t)$ is time-varying signal voltage.

According to the theory of electronics, it can be obtained that the variation of $V_{\mathrm{ms}}$ with time is proportional to the change rate of the AE signal energy. The total energy $E$ of the AE signal during the time $t_{1} \sim t_{2}$ can be expressed as follows:

$$
E \propto \int_{t_{1}}^{t_{2}}\left(V_{\mathrm{rms}}\right)^{2} d t=\int_{t_{1}}^{t_{2}} V_{\mathrm{ms}} d t .
$$

The acoustic emission energy rate is the sum of the absolute energy of the acoustic emission events per unit time, reflecting the elastic energy released by the internal cracks or expansion of the rock.

The experiment considers the influence of the distance and location of the fracture source and the sensors. Without affecting the observation, two sensors are used to collect the acoustic emission signals of the rock fracture process at the same time. Through analyzing the characteristics of acoustic emission parameters of two channels during the experiment, it is concluded that the distance and location of the fracture source and the sensors in the experiment will have, to a certain degree, an impact on the acoustic emission received, but there are similarities in the characteristics of the $\mathrm{AE}$ signals received by sensors at different locations. It is found that the acoustic emission absolute energy rate and the event rate curves of two channels are approximately the same, and the values are different. When calculating the absolute energy of acoustic emission, with the choice of a channel, or two channels, the energy of which only exists in the numerical differences, there is no regular change. Therefore, during energy calculation, only one channel is selected for energy statistics. The selection is based on a channel with a relatively large absolute energy rate peak.

3.2. Frequency-Domain Information Extraction. The AE signal is a nonstationary signal. Fast Fourier transform (FFT) is a classical spectral analysis method for analyzing nonstationary signals [28]. The spectral characteristics of the acoustic emission signal produced by the rock can characterize the rock state, rock structure, and mechanical properties. Select the sample HSHG-2 to conduct acoustic emission number 24926 of waveform to illustrate the waveform file frequencydomain parameters (frequency, dominant-frequency amplitude) extraction process; the specific process is as follows:

(1) Using MATLAB to write the program, extract the original acoustic emission waveform signal, as shown in Figure 3(a).

(2) The filtered waveform is subjected to fast Fourier transform to obtain a two-dimensional spectrum, as shown in Figure 3(b).

By changing the acoustic emission waveform from the time domain to the frequency-domain, the frequency distribution characteristic can be obtained, and the dominantfrequency is defined as the frequency corresponding to the maximum amplitude in the spectrum [11]. Observing the waveform spectrum, it can be seen that the dominantfrequency of acoustic emission waveform signal is $38.09 \mathrm{kHz}$.

\section{The Relation on Rock Fracture Scale and Acoustic Emission Time-Frequency Information}

4.1. Quantitative Characterization of Crack Scales in Rock Fracture Process. Some studies have shown that the crack scales of rock fracture process can be indirectly characterized by the $b$ value of acoustic emission. In 1941, Gutenberg and Richer proposed statistical relations between magnitude and frequency of earthquakes in the study of worldwide seismicity, namely, the well-known G-R relation [29]:

$$
\log N=a-b M,
$$

where $M$ is the magnitude and $N$ is the number of earthquakes. $a$ and $b$ are constants, where $b$ is a function of relative magnitude distribution of acoustic emission. Therefore, $b$ value is also a function of crack propagation scale, and actually characterizes the distribution of the number of microcracks with the microcrack scale. The $b$ value not only is a statistical analysis parameter, but also has a direct physical 


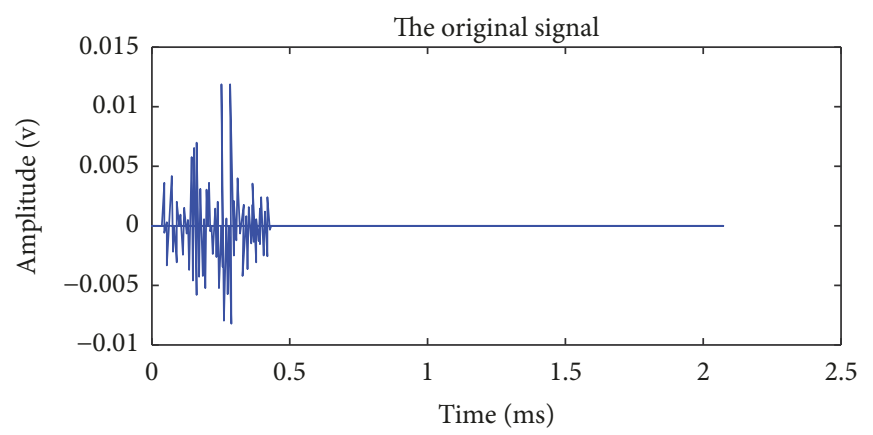

(a) The original AE signal

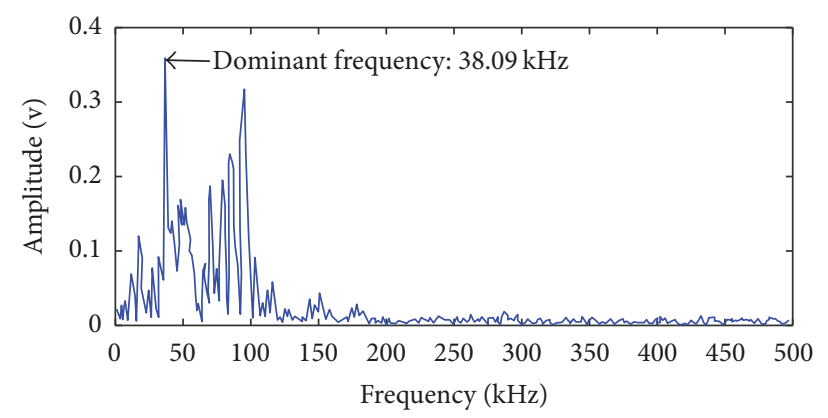

(b) Spectrum map

FIGURE 3: The extraction process of AE dominant-frequency.

meaning. The increase of $b$ value means the proportion of small-scale cracks increases. The stagnancy of $b$ value means that the crack scales distribution is constant. The decrease of $b$ value means the proportion of large-scale cracks increases.

The calculation method of acoustic emission $b$ value is as follows. First, determine the acoustic emission event magnitude (there is no uniform definition on the acoustic emission magnitude at present, and this paper defines the magnitude $M$ of the acoustic emission event is the logarithm of the acoustic emission energy at the base of 10 with 0.5 magnitude interval). The cumulative frequency is selected for the statistical $N$ value, and the acoustic emission $b$ value can be calculated by the linear least squares method. In order to avoid the error of $b$ value calculation since the number of acoustic emission events is too scarce in a certain period of time, 1000 acoustic emission events are taken as a set of data to calculate the $b$ value, and 100 acoustic emission events are set as the sliding window and the window ending time is chosen as the moment of $b$ value to get the change process of $b$ value with time (strain). Based on the analysis of Figure 5 , it is found that there are only small-scale fractures in stages I, II and III, and large-scale fractures in stage IV. Therefore, the average $b$ value in stages I, II and III is taken as the overall $b$ value of small-scale fractures, and the average $b$ value of IV stage $b$ value is taken as the overall $b$ value of large-scale fractures, as shown in Table 1 with $b$ value of different rock samples. It can be seen from the table that $b$ value of small-scale crack is 0.6648 and $b$ value of large-scale crack is 0.4028 , which conforms to the relation between $b$ value and crack scales. Therefore, $b$ value can be used to characterize the scales of crack.

\subsection{The Relation of Rock Fracture Scale and Acoustic Emission} Energy. In Section 4.1, the $b$ value of acoustic emission was used to quantitatively characterize the crack scale in the granite fracture process. On the basis of this, the correlation between the $b$ value of the acoustic emission and the absolute energy of the granite fracture process was analyzed. Then the relation between $\mathrm{AE}$ absolute energy and crack scales is established according to $b$ value. Figure 4 shows the correlation between the $b$ value of acoustic emission and the absolute energy during the fracture process of different granite samples. It can be seen from the figure that the
TABLE 1: $b$ value of small- and large-scale cracks.

\begin{tabular}{lcc}
\hline Sample number & \multicolumn{2}{c}{$b$ value } \\
\hline HSHG-1 & Small-scale & Large-scale \\
HSHG-2 & 0.737 & 0.445 \\
HSHG-3 & 0.615 & 0.319 \\
HSHG-4 & 0.654 & 0.433 \\
HSHG-5 & 0.688 & 0.308 \\
Average & 0.630 & 0.509 \\
\hline
\end{tabular}

absolute acoustic emission energy is the maximum value when the $b$ value of acoustic emission is the minimum value, which shows that the large-scale cracks are dominant with relatively high absolute energy; with the increase of $b$ value of acoustic emission, the absolute energy of acoustic emission decreases sharply and becomes stable. According to the physical meaning that the $b$ value of acoustic emission increases, it means that the proportion of small-scale cracks increases. Therefore, corresponding to the increase of the proportion of small-scale cracks, the absolute energy of acoustic emission decreases sharply and then becomes stable.

Based on the above analysis, the relation between the crack scales and the absolute energy of acoustic emission can be obtained. For large-scale cracks, the absolute energy of acoustic emission is relatively high, while the absolute energy of acoustic emission corresponding to small-scale cracks is often low.

Figure 5 shows the variation curve of the load, the acoustic emission event rate, and the absolute energy rate changing with time in granite fracture process. It can be seen from the figure that the evolution of the acoustic emission event rate in granite fracture process can be divided into four stages: (I) slow ascent period, the acoustic emission event rate is maintained at a relatively low value and rises slowly; (II) accelerated ascent period, the acoustic emission event rate rises sharply; (III) accelerated descent period, the acoustic emission event rate drops quickly; (IV) quiet period, the acoustic emission event rate was kept at a small constant value until the rock ruptured. At the same time, it can be 


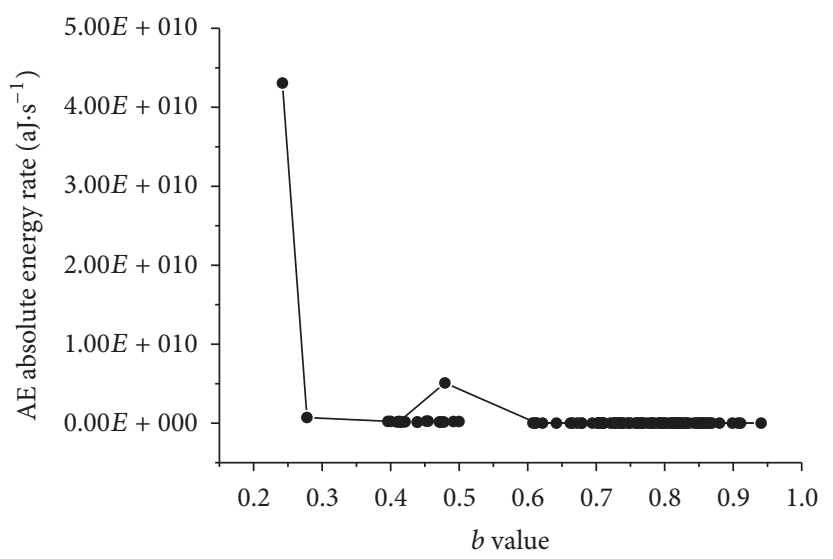

(a) HGHG-1

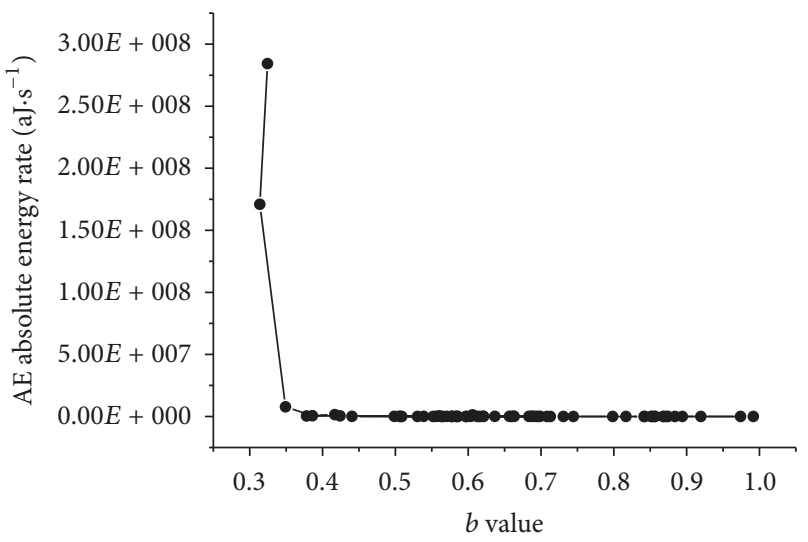

(b) HSHG-2

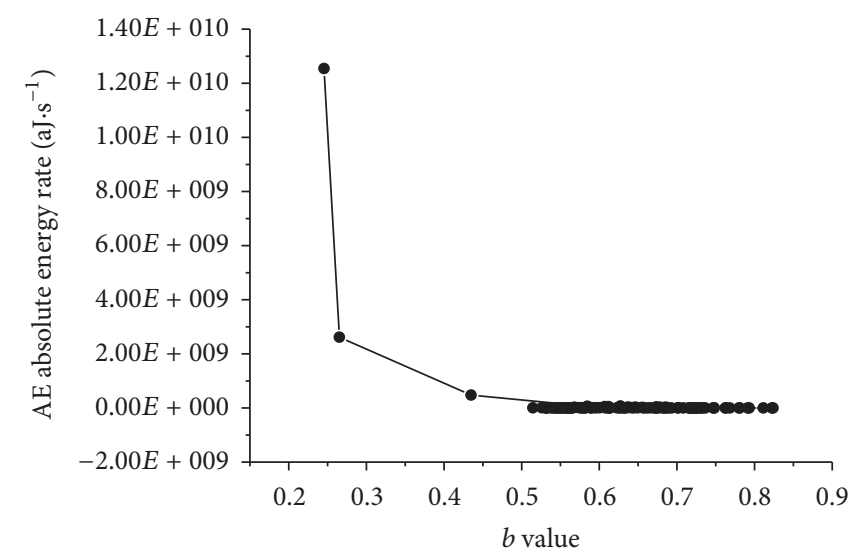

(c) HSHG-5

FIGURE 4: Correlation diagram between $\mathrm{AE} b$ value and absolute energy in the granite fracture process.

seen that the absolute energy rate of acoustic emission is maintained at a low level in the stages of I and II, which release basically no energy during the whole process. In stage III, the acoustic emission energy rate starts to rise slowly with a small amount of energy release; in stage IV, the acoustic emission energy rate rises sharply during the quiet period, which means that the accelerated energy release at this stage. The above analysis shows that the acoustic emission event rate falls from the peak to a small constant value of about 10 beats/second before the granite fracture happens, which can be called the quiet period of acoustic emission. During the period, the acoustic emission energy accelerated releasing which results in the phenomenon that the acoustic emission event rate is calm while the energy rate is not. During the quiet period, correlation analysis results of the acoustic emission characteristics and the acoustic emission event and energy rate are consistent. The acoustic emission event rate is maintained at a low constant value of about 10 times/sec and the acoustic emission energy rate is relatively high.

For the quiet period of acoustic emission before rock burst, Yin et al. [30], Liu et al. [4], and Zhao et al. [31] pointed out that there is an objective phenomenon of acoustic emission calm period in acoustic emission test under rock uniaxial compression. For the reasons of the absence of acoustic emission events before rock failure, Li et al. [32] explained that one reason is that a large number of cracks are accumulated before failure, which make the acoustic emission radiation wave attenuate seriously and even be blocked by the crack; another reason is that when the destruction is approaching, the surface of the rock has been seriously damaged and become uneven. The coupling between the acoustic emission sensor and the rock surface is close to disengagement, so that the sensor can not receive the waveform normally. The author believes that the above two aspects only explain the physical factors of propagation and reception of acoustic emission waves. After analysis, it is found that when the granite enters into the acoustic emission calm period before rupture, the acoustic emission event rate is small while the absolute energy rate rises sharply. This is mainly due to the rapid expansion of a large number of smallscale cracks which forms large-scale cracks before granite fracture. The number of cracks decreases continuously during the formation of large-scale cracks, but the scale increases sharply, resulting in the decrease of the acoustic emission events of unit time and the dramatic increase of energy released, which shows the quiet period of acoustic emission event rate, while the absolute energy rate of acoustic emission is not calm. 


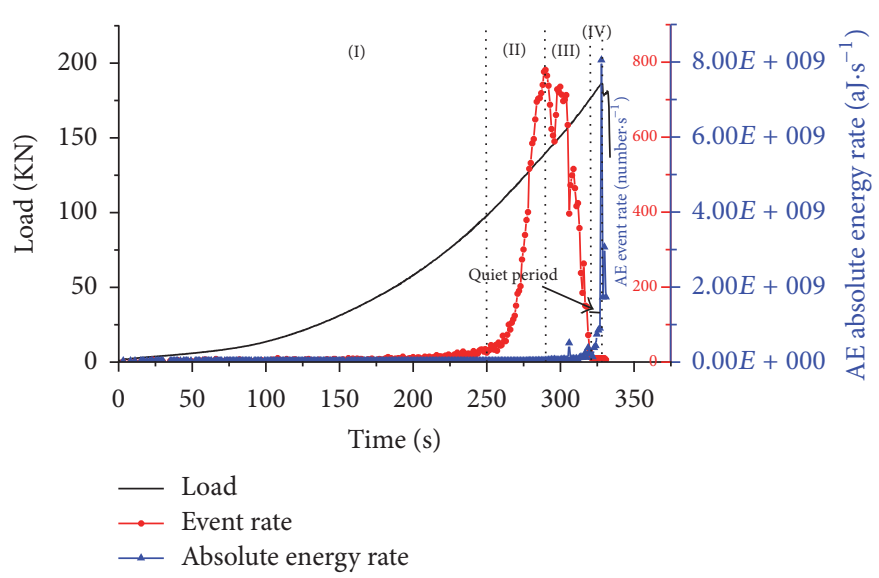

(a) HSHG-2

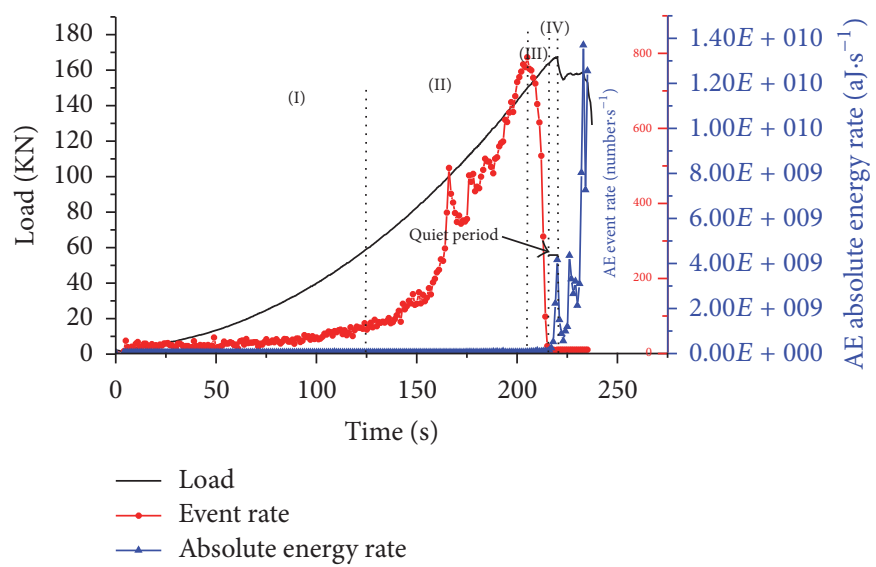

(b) HSHG-5

FIGURE 5: The change curve of load, AE event rate, and the absolute energy rate during the failure process of granite.

TABLE 2: Characteristic of time-domain parameters of AE quiet period of granite.

\begin{tabular}{lcr}
\hline Sample number & & AE quiet period \\
HSHG-1 & AE event rate times/s & Energy release percentage\% \\
HSHG-2 & 10 & 80.05 \\
HSHG-3 & 10 & 81.33 \\
HSHG-4 & 10 & 93.31 \\
HSHG-5 & 10 & 75.46 \\
Average & 10 & 89.22 \\
\hline
\end{tabular}

Table 2 shows the characteristics of event rate and energy rate of the granite acoustic emission in the quiet period. The acoustic emission event rate is a constant value of the event rate during the quiet period. The energy release proportion of acoustic emission is the ratio of the release of energy during the acoustic emission quiet period and the total release of energy during the rock fracture process. It can be found that the acoustic emission event rate is maintained at 10 times/s during the quiet period and about $83.87 \%$ energy of the whole fracture process is released in a very short period of time.
According to the results of Table 2, during the quiet period of acoustic emission before the granite fracture, the acoustic emission event rate is 10 times/s, and about $83.87 \%$ energy of the whole fracture process of granite is released in a very short period of time. This means that, before the granite fracture, the acoustic emission event rate is low, but the fracture scale is large and the energy release is high. In summary, the acoustic emission energy can represent the crack scale in the rock fracture process: when the acoustic emission energy is high, the corresponding acoustic emission 
event fracture scale is large, and when the acoustic emission energy is low, the corresponding acoustic emission event fracture scale is small. According to the principle of acoustic emission monitoring, some of the energy is released in the form of elastic waves during the crack propagation process. The acoustic emission signals can be received by sensors. Thus, the acoustic emission energy is related to the energy released at the time of crack propagation and is proportional to it. According to the theory of fracture mechanics [33], considering the new crack at the length of $2 \mathrm{c}$ unit width and the new surface energy of the crack generation process being proportional to $2 c$, the energy released at this time is equal to the newly added surface energy of crack expansion. Thus, the energy released by the crack propagation process is proportional to $2 c$; that is, the acoustic emission energy is proportional to the crack scale [34].

4.3. The Relation of Rock Fracture Scale and Acoustic Emission Dominant-Frequency. According to Figure 3(b), it can be seen that the frequency range of the acoustic emission signal is $0 \sim 500 \mathrm{kHz}$ after FFT transformation. Considering the distribution characteristics of the dominant-frequency of the granite acoustic emission signal, three frequency bands of $0 \sim 128 \mathrm{kHz}, 128 \sim 256 \mathrm{kHz}$, and $256 \sim 500 \mathrm{kHz}$ are called low frequency, medium frequency, and high frequency, respectively. When the dominant-frequency statistical analysis is carried out, the acoustic emission frequency of the mechanical vibration during the rock loading process is basically the low frequency component that is lower than $30 \mathrm{kHz}$ according to the spectral transformation result [35]. Therefore, the low frequency (mechanical vibration) and low amplitude (electromagnetic signal collected by instruments) are removed; then, the statistical range of low frequency is $30 \sim 128 \mathrm{kHz}$, the medium frequency is $128 \sim 256 \mathrm{kHz}$, and the high frequency is $256 \sim 500 \mathrm{kHz}$.

All the acoustic emission waveforms of the experiment are processed and statistically analyzed to obtain the dominant-frequency characteristics of the acoustic emission signals in the rock fracture process. According to the idea of acoustic emission energy characterizing the energy of acoustic emission signal from the perspective of time domain, the corresponding relation between acoustic emission energy and dominant-frequency is analyzed, and the dominantfrequency characteristics of high and low acoustic emission energy signals, respectively, are studied. Based on the relation between the energy and the fracture scale above, the relation between the fracture scale and the dominant-frequency is discussed, that is, the dominant-frequency characteristics corresponding to the small and the large-scale fracture.

Figure 6 shows the correlation between the absolute energy and the dominant-frequency of the acoustic emission during the granite fracture process. It can be seen from Figure 6 that there are three absolute high-energy peak values (as shown in the figure) in the low frequency region of the dominant-frequency of acoustic emission signal, the corresponding main frequencies of which are $41 \mathrm{kHz}, 92 \mathrm{kHz}$, and $103 \mathrm{kHz}$, that is, the low frequency high-energy signal. The dominant-frequency distribution of the acoustic emission signal with low absolute energy is about $34 \sim 115 \mathrm{kHz}, 150 \mathrm{kHz}$,
$175 \mathrm{kHz}$, and $275 \mathrm{kHz}$. In general, the dominant-frequency of the acoustic emission signal corresponding to low energy is distributed among the low, medium, and the high frequency region, so three types of acoustic emission signals, low frequency low energy, medium frequency low energy, and high frequency low energy, appeared. According to the proportional relation between the fracture scale and the energy in the process of granite fracture, it can be concluded from further analysis that the dominant-frequency of high-energy signal corresponding to the large-scale fracture is mainly concentrated in the low frequency region, which is $41 \mathrm{kHz}$, $92 \mathrm{kHz}$, and $103 \mathrm{kHz}$, respectively; the dominant-frequency distribution of the acoustic emission corresponding to the small-scale fracture is within the range of low frequency (34 $115 \mathrm{kHz})$, medium frequency $(150 \mathrm{kHz}, 175 \mathrm{kHz})$, and high frequency $(275 \mathrm{kHz})$.

From the relation between the acoustic emission dominant-frequency and the fracture scale, it can be found from the point of view of dominant-frequency that, in the low frequency region, the dominant-frequency characteristics corresponding to the small and large-scale fracture coexisted, which makes it difficult to distinguish the characteristics of acoustic emission signals corresponding to different fracture scales. Wan and Zhou argue that the high frequency of the acoustic emission signal corresponds to the small-scale fracture, and the low frequency corresponds to the largescale fracture; that is, the fracture scale and the frequency are inversely proportional [36]. The results show that, in the low frequency region, the high-energy low frequency acoustic emission signals correspond to large-scale fractures. In the medium and high frequency regions, the energy of acoustic emission signals is low, and the medium frequency low energy and high frequency low energy acoustic emission signals correspond to small-scale fractures. Based on the above analysis, it can be seen that the large-scale fractures correspond to the low frequency and high-energy acoustic emission signals, and the small-scale fractures correspond to the medium and high frequency low energy acoustic emission signals. Only in the case that the rock fracture scale and energy were proportional, the conclusion that the fracture scale of granite and the dominant-frequency are inversely proportional can be drawn.

Therefore, the use of single parameter (such as dominantfrequency) of acoustic emission usually can not accurately characterize the relation between rock fracture scale and acoustic emission information; therefore, the acoustic emission energy is needed to combine with the dominantfrequency to determine the acoustic emission characteristics corresponding to different fracture scales.

\subsection{Relation between Rock Fracture Scale and Acoustic Emis-} sion Time-Frequency Information. Based on the discussion of the relation between rock fracture scale and acoustic emission time-frequency information (energy, frequency) based on Sections 4.1 and 4.2 , the time-frequency characteristics of acoustic emission signals corresponding to different fracture scales are obtained. Considering the two parameters of acoustic emission energy and dominant-frequency, it can be concluded that the fracture scale and the acoustic 


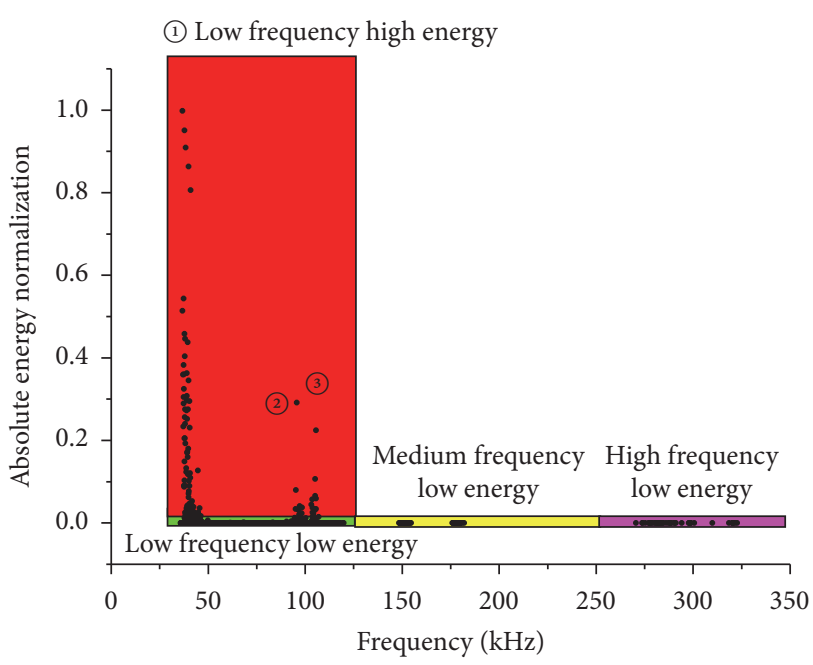

(a) HSHG-1

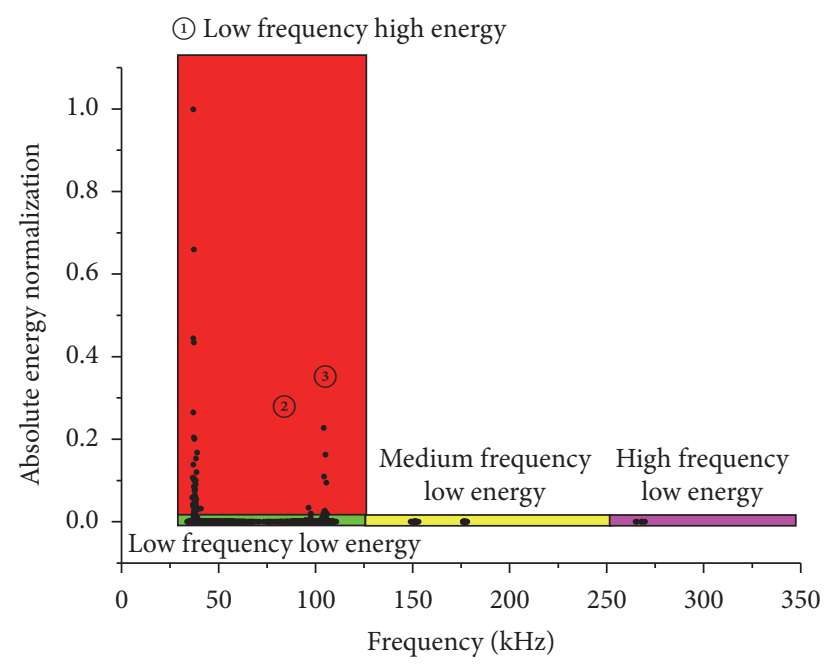

(b) HSHG-2

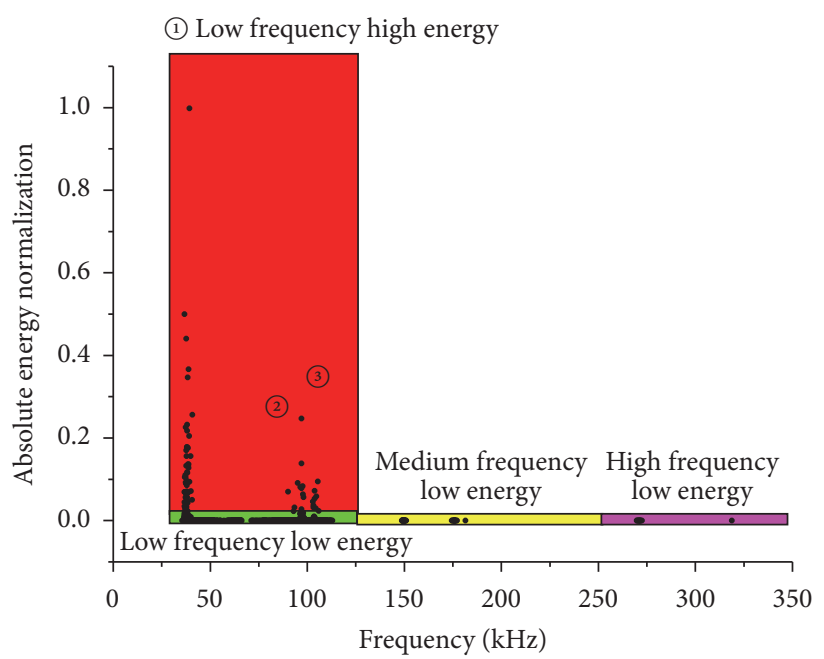

(c) HSHG-4

FIGURE 6: The correlation diagram of dominant-frequency and energy in the process of granite fracture.

emission energy of the rock fracture process are proportional and are inversely proportional to the acoustic emission dominant-frequency. Low frequency high-energy acoustic emission signals often correspond to large-scale fractures; high frequency low energy acoustic emission signals often correspond to small-scale fractures. The studies of Santis and Tomor indicate that high-amplitude waveform signals are usually concentrated in low frequency regions, which forebodes the high-energy release [37]. It is consistent with the analysis results of large-scale fractures corresponding to slow-frequency high-energy signals.

\section{Theory and Application of}

\section{the Relation between the Acoustic Emission Time-Frequency Information and Rock Fracture Scale}

5.1. Analysis of the Relation between Rock Fracture Scale and Acoustic Emission Time-Frequency Information. Based on the above analysis of time-frequency characteristics of acoustic emission signals during the rock fracture process, it is found that there is a correspondence between the type of acoustic emission signals and the fracture scale, as shown in Table 3. It should be pointed out that the statistics of absolute energy of acoustic emission refers to the $b$ value statistical methods. In Figure 5, the average absolute energy in stages I, II, and III is taken as the total absolute energy of small-scale cracks, and the average absolute energy in phase IV is taken as the total absolute energy of large-scale cracks. Large-scale cracks in granite fracture process correspond to the low frequency and high-energy acoustic emission acoustic signals, whose dominant frequencies are often located in the low frequency region of $41 \mathrm{kHz}, 92 \mathrm{kHz}$, and $103 \mathrm{kHz}$ with relatively high absolute energy, about $1.15 \times 109$ aJ; while small-scale cracks show medium/high frequency and low energy features, the dominant-frequency is often located in the medium/high frequency regions of $150 \mathrm{kHz}, 175 \mathrm{kHz}$, and $275 \mathrm{kHz}$ with relatively low absolute energy, about $9.20 \times 106$ aJ. On the 
TABLE 3: Corresponding acoustic emission characteristics of different fracture scale cracks.

\begin{tabular}{lcccc}
\hline Crack scale & $b$ value & Types of AE signal & Dominant frequency/khz & Absolute energy/aJ \\
\hline Large scale cracks & 0.6648 & Low frequency and high energy & $\begin{array}{c}\text { Low frequency } \\
(41 \mathrm{khz}, 92 \mathrm{khz} \text {, and } \\
103 \mathrm{khz})\end{array}$ & High energy $\left(1.15 \times 10^{9}\right)$ \\
Small scale cracks & 0.4028 & Medium/high frequency and low energy & $\begin{array}{c}\text { Medium/high frequency } \\
(150 \mathrm{khz}, 175 \mathrm{khz}, \text { and } \\
\end{array}$ & Low energy $\left(9.20 \times 10^{6}\right)$ \\
\hline
\end{tabular}

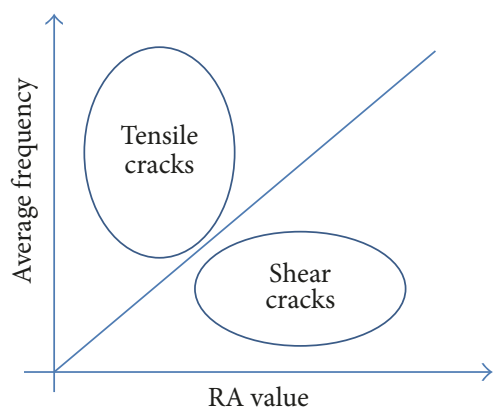

FIGURE 7: Conventional crack classification [27].

whole, the acoustic emission signals of large-scale fracture in the granite rupture process is characterized by low dominantfrequency and high-energy, and the small-scale fracture of which is characterized by low energy and medium/high frequency.

Based on this, the energy and the dominant-frequency of the AE parameters can reflect the scale of cracks inside the material. In practical applications, the two parameters should be combined to better describe the acoustic emission characteristics corresponding to different crack sizes, and then put forward the evaluation method of rock fracture scales based on the acoustic emission signal energy and frequency. According to the correlation analysis between rock fracture scales and acoustic emission time-frequency information (energy and frequency), it is found that the rock fracture scales are proportional to the energy of acoustic emission signals and inversely proportional to the dominantfrequency of acoustic emission signals. In general, acoustic emission signals with low dominant-frequency and highenergy generally represent the generation or development of large-scale cracks. On the contrary, high dominant-frequency and low energy are the generation or development of smallscale cracks.

It has been shown that acoustic emission signals with high RA values and low AF values correspond to shear fractures. Meanwhile, acoustic emission signals with low RA values and high AF values correspond to extension fractures. Based on this, the rock fracture mode analysis method based on acoustic emission RA-AF values is established, as shown in Figure 1. Therefore, with reference to the classification method in Figure 7, the paper sets up a method to classify the crack scales in the rock fracture process based on the dominant-frequency and energy of AE, as shown in Figure 8.

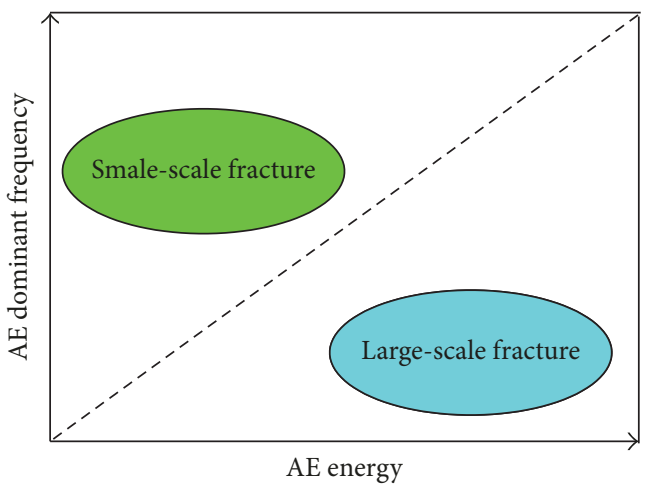

FIGURE 8: Rupture scale classification diagram.

5.2. Application Example. Based on the theory of rock fracture scale and acoustic emission information (energy, dominant-frequency) in Section 5.1, the crack scales of change of granite uniaxial compression and rupture process is analyzed. Figure 9 shows the acoustic emission energy-dominant-frequency scatter plot for the whole process of typical granite specimen fracture. According to the crack classification map of the rock fracture process, it can be seen from Figure 8 that the rock fracture process mainly consists of small-scale cracks, resulting in a small amount of large-scale cracks.

In order to better express the characteristic distribution of the acoustic emission parameters and then reflect the crack types, the concept of mathematical stochastic data probability density function is selected to perform distribution density calculation on the energy-dominant-frequency parameters, as shown in Figure 10. Figure 10 depicts the density distribution diagram of the acoustic emission energy-dominantfrequency at each stage of the granite fracture process. The red area represents the densest area, that is, the core area of data distribution; the blue area is free of data point distribution so the density is 0 , and the transitional region from red to blue area represents a certain amount of data. By analyzing the density distribution diagram of the acoustic emission energy-dominant-frequency of the granite fracture process, the evolutionary characteristics of fracture scale of each stage of the rock fracture process can be found.

(1) Compression stage: the red area in the figure shows that the acoustic emission signal is mainly concentrated in the low frequency and low energy area, and 


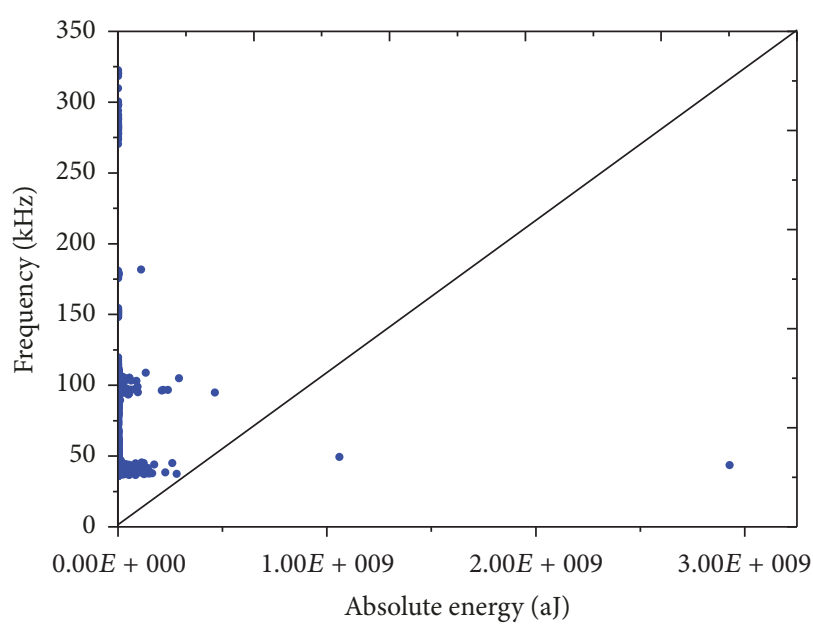

(a) HSHG-1

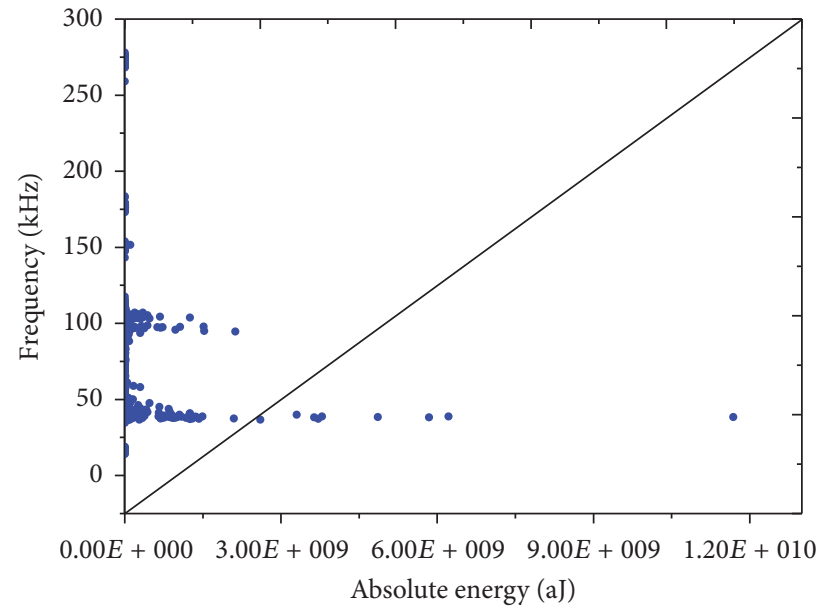

(b) HSHG-5

FIGURE 9: Acoustic emission energy during the granite rupture process dominate frequency scatter plot.

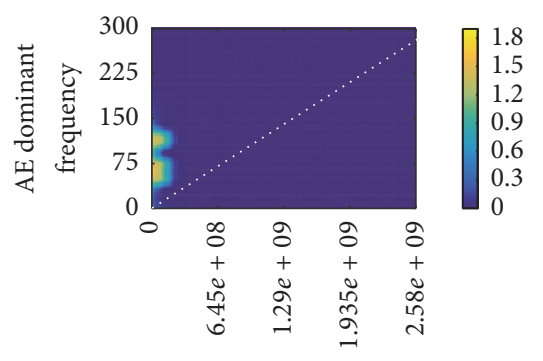

AE absolute energy

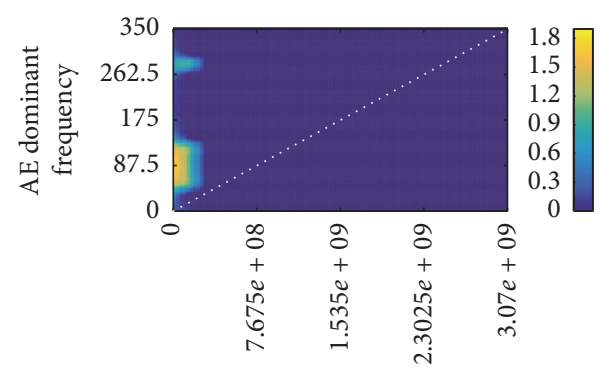

AE absolute energy

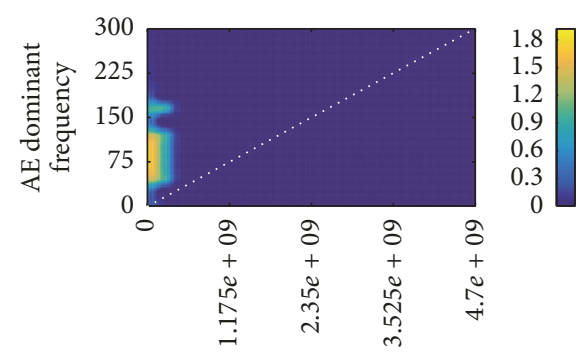

AE absolute energy

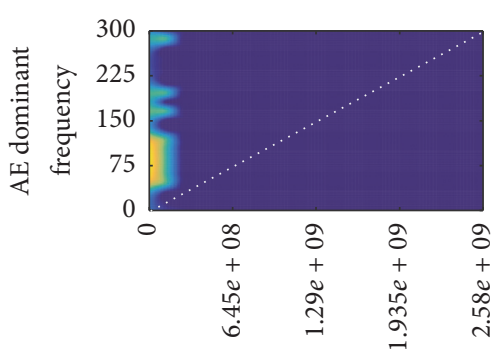

AE absolute energy

(a) HSHG-2

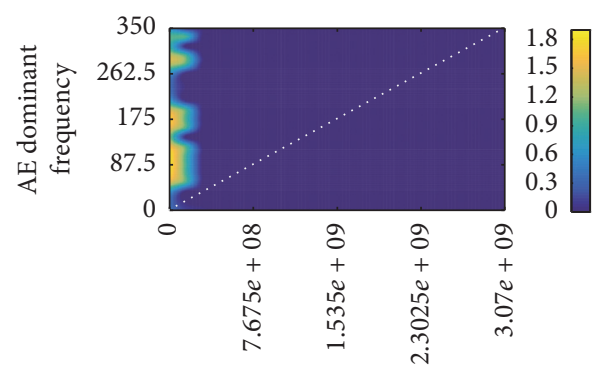

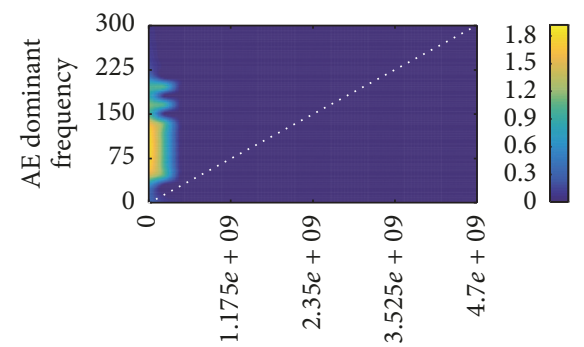

AE absolute energy
AE absolute energy

(b) HSHG-4

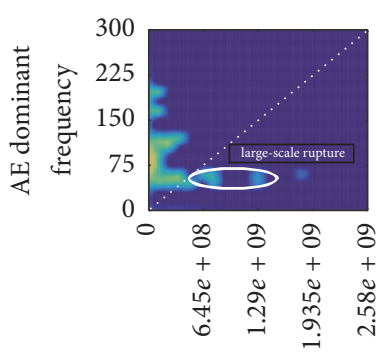

AE absolute energy

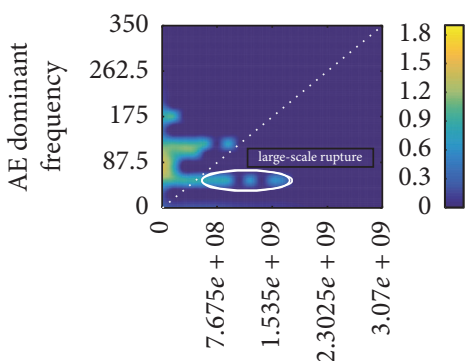

AE absolute energy

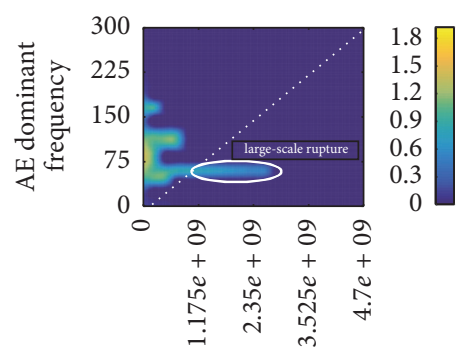

AE absolute energy

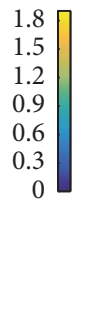

1.5
1.2
0.9
0.6
0.3
0

(c) HSHG-5

FIGURE 10: Acoustic emission energy-frequency distribution of granite during rupture. 
the acoustic emission demonstrates low frequency and low energy characteristics, which means that small internal scale fracture happens in this stage.

(2) Elastic stage: the red area is also concentrated in the low frequency region but larger than the previous stage, and in the medium and high frequency region, the transitional region from red to blue area appears. It manifests that the acoustic emission signal is mainly concentrated in the low frequency and low energy region, but there are medium and high frequency low energy acoustic emission signals, which means that the components of acoustic emission frequency are complex in elastic phase. The range of acoustic emission frequency widened, but the energy is low, resulting in small-scale fractures.

(3) Plastic stage: the red data core area is getting smaller but is still in the low frequency low energy area. The transitional region of high frequency ranges from red to blue area and disappears and transfers to low frequency region, so the distribution area increases, especially transitional region from red to blue area in the low frequency high-energy range. It is shown that the high frequency low energy acoustic emission signal disappears during the plastic phase, which is due to the high attenuation of the high frequency signal in the propagation process [38], so the acoustic emission sensors can not receive the signal. The distribution of AE signal dominant-frequency is in the medium and low frequency region, but as the acoustic emission energy increases, the emergence of low frequency high-energy acoustic emission signal could happen. This means that, in the plastic phase, there are mainly small-scale fractures within the rock, but the fracture scale changes leading to complete destruction eventually.

Through the above analysis, by using the acoustic emission energy-frequency density cloud map, the evolution characteristics of the rupture scale at different stages of rock fracture process under uniaxial compression can be found. In the compression stage, small internal scale fracture happens; in the elastic phase, there are also small-scale fractures, but, comparing with the compression stage, the range of acoustic emission frequency widened, which means that the smallscale fracture modes are getting complex. In the plastic stage, there are mainly small-scale fractures within the rock as well as the occurrence of large-scale fractures. The above results are consistent with the analysis of the evolution characteristics of the rupture scale of rock fracture process under uniaxial compression in literature [39]. Therefore, based on the acoustic emission time-frequency information (energy, frequency), the theory and method of evaluation of rock fracture scale can accurately describe the variation characteristics of the crack scale during the rock fracture process and provide a way to quantitatively characterize the crack scales of the rock fracture process.

\section{Discussion}

6.1. Physical Significance of Experimental Results. From the analysis results of this paper, it is found that the acoustic emission dominant-frequency of the large-scale cracks in the granite fracture process is often located in the low frequency region and the acoustic emission energy is relatively large. The acoustic emission dominant-frequency of the small-scale cracks is usually located in the middle and high frequency regions with relatively low AE energy. Namely, the larger the rock fracture scale, the smaller the acoustic emission frequency and the greater the acoustic emission energy. Therefore, it can be considered that the crack scales in the rock fracture process are one of the main factors that influence the dominant-frequency and energy of acoustic emission. As the crack scale increases, the dominant-frequency of the AE signal gradually decreases and the energy gradually increases. This result is in agreement with the researches of Ohnaka and Mogi [40, 41]. The decrease of AE frequency and increase of AE energy before rock failure are a sign of the crack scale becoming larger. The physical mechanism is that when the fracture occurs, the scale of the fracture source increases, so the interaction of cracks is significantly enhanced, leading to dominant-frequency reduction and energy increase.

Based on the above analysis, the author believes that the increase of crack scale in the rock fracture process results in scale effect which causes the dominant-frequency of acoustic emission signal to decrease and acoustic emission energy to increase. As a result, different crack scales correspond to different acoustic emission signal types. Therefore, the variation of $\mathrm{AE}$ frequency and energy in rock fracture process is significant and may provide the monitoring basis for predicting the occurrence of rock catastrophe (rock burst).

6.2. Discussion on the Application of Experimental Results. With the increase of energy demand and the intensity of mining, the resources in the shallow areas are declining, and the mines both at home and abroad have entered the state of deep resource exploitation successively. According to incomplete statistics, there are over 80 ultra-deep (more than 1000 kilometers) metal mines in the world, most of which are in South Africa. The mining depth of vast majority of gold mines in South Africa exceeds $1000 \mathrm{~m}$. In addition, some nonferrous metal mines in Canada, the United States, and Australia also have a depth of over $1000 \mathrm{~m}$. It is estimated that, in the next 20 years, many coal mines in our country will enter the depth of $1000 \sim 1500 \mathrm{~m}$. At the same time, China's metal and nonferrous metal mines will enter a depth of 1000 2000 m. What clearly distinguishes between deep and shallow mining is the special environment in which deep rocks are located, that is, the complex mechanical environment of the three highs and one disturbance, which includes the high ground stress, high temperature, high karst water pressure, and mining disturbances. After entering the deep mining, under the influence of three highs and one disturbance, the geotechnical environment of the deep engineering surrounding rock has undergone great changes, so that the surrounding rock of deep roadway shows its unique mechanical characteristics. And with the 
complexity of the environment, rock dynamic disasters such as rock bursts have become more and more serious, posing a huge threat to the safe and efficient exploitation of deep resources.

It is well known that any destruction takes place from quantitative change to qualitative change, that is, the process of rock mass from minor fracture to destruction, so does the occurrence of rock burst. In essence, rock burst is the process of primary fissure closure, crack formation, expansion, and penetration. In this process, the scale of crack increases continuously which reflects in the decrease of the dominantfrequency and the increase of energy of acoustic emission. For deep rock burst disaster, the rock burst process can also be monitored by acoustic emission technology in real time. The analysis of the change of acoustic emission dominantfrequency and energy in the rock burst process plays an extremely important role in the research of rock burst mechanism and the prediction of rock burst which has a wide range of engineering application value.

\section{Conclusions}

(1) A single acoustic emission parameter can not accurately reflect the relation between fracture scale and acoustic emission information. It is necessary to combine the acoustic emission time domain and frequency-domain characterization parameters, that is, using acoustic emission energy and dominant-frequency to determine the acoustic emission characteristics corresponding to different fracture scales. Large-scale fracture corresponds to low frequency highenergy acoustic emission signal, and small-scale fracture corresponds to high frequency low energy acoustic emission signal.

(2) The acoustic emission energy and dominantfrequency can reflect the internal crack scale of the rock, and the evaluation method of the rock rupture scale based on the energy and frequency of the acoustic emission signal is proposed. The fracture scale of the rock fracture process is proportional to the acoustic emission energy and is inversely proportional to the acoustic emission dominant-frequency. In general, a low frequency, high-energy acoustic emission signal usually represents the generation or development of large-scale cracks; on the contrary, if it is a high frequency, low energy value, it is a small-scale crack.

(3) The evolution law of the fracture scale under uniaxial compression fracture scale shows that the evaluation theory and method of rock fracture scale based on the acoustic emission time-frequency information (energy, frequency) can accurately describe the variation characteristics of the crack scale during the rock fracture process and provide a way to quantitatively characterize the crack scales of the rock fracture process.

\section{Conflicts of Interest}

The author declares that there are no conflicts of interest regarding the publication of this paper.

\section{Acknowledgments}

The author wishes to acknowledge the support of National Key Research and Development Program (2016YFC0600901), Research Fund of the State key Laboratory of Coal Resources and Safe Mining (SKLCRSM18KF021), and the Fundamental Research Funds for the Central Universities (Grant no. 2018YZ01).

\section{References}

[1] L. Dong, J. Wesseloo, Y. Potvin, and X. Li, "Discrimination of mine seismic events and blasts using the fisher classifier, naive bayesian classifier and logistic regression," Rock Mechanics and Rock Engineering, vol. 49, no. 1, pp. 183-211, 2016.

[2] L. Dong, J. Wesseloo, Y. Potvin, and X. Li, "Discriminant models of blasts and seismic events in mine seismology," Int. J. Rock Mech. Min. Sci, vol. 86, pp. 282-291, 2016.

[3] R. Zhang, H. Xie, J. Liu, J. Deng, and Q. Peng, "Experimental study on acoustic emission characteristics of rock failure under uniaxial multilevel loadings," Yanshilixue Yu Gongcheng Xuebao/Chinese Journal of Rock Mechanics and Engineering, vol. 25, no. 12, pp. 2584-2588, 2006.

[4] J. Liu, S. Xu, Y. Li, L. Dong, and J. Wei, "Studies of AE timespace evolution characteristics during failure process of rock specimens with prefabricated holes," Yanshilixue Yu Gongcheng Xuebao/Chinese Journal of Rock Mechanics and Engineering, vol. 31, no. 12, pp. 2538-2547, 2012.

[5] J. Xu, H. Wu, L. Lu, H. Yang, and H. Tan, "Experimental study of acoustic emission characteristics during shearing process of sandstone under different water contents," Chinese Journal of Rock Mechanics and Engineering, vol. 31, no. 5, pp. 914-920, 2012.

[6] C. Su, B. Gao, H. Nan, and X. Li, "Experimental study on acoustic emission characteristics during deformation and failure processes of coal samples under different stress paths," Yanshilixue Yu Gongcheng Xuebao/Chinese Journal of Rock Mechanics and Engineering, vol. 28, no. 4, pp. 757-766, 2009.

[7] M. Cai, H. Morioka, P. K. Kaiser et al., "Back-analysis of rock mass strength parameters using AE monitoring data," International Journal of Rock Mechanics and Mining Sciences, vol. 44, no. 4, pp. 538-549, 2007.

[8] X. B. Yu, Q. Xie, X. Y. Li, Q. R. Wang, and Z. P. Song, "Acoustic emission of rocks under direct tension, brazilian and uniaxial compression," Chinese Journal of Rock Mechanics and Engineering, vol. 26, no. 1, pp. 137-142, 2007.

[9] S. Li, X. Yin, Y. Wang, and H. Tang, "Studies on acoustic emission characteristics of uniaxial compressive rock failure," Chinese Journal of Rock Mechanics and Engineering, vol. 23, no. 15, pp. 2499-2503, 2004.

[10] Y. J. Yang, D. C. Wang, M. F. Guo, and B. Li, "Study of rock damage characteristics based on acoustic emission tests under triaxial compression," Chinese Journal of Rock Mechanics and Engineering, vol. 23, pp. 98-104, 2014.

[11] X. N. Jia, Experimental study on acoustic emission Eigenfrequency spectrum features of strain bursts, China University of Mining and Technology, Beijing, China, 2013.

[12] Y. Gong, M. He, Z. Wang, and Y. Yin, "Research on timefrequency analysis algorithm and instantaneous frequency precursors for acoustic emission data from rock failure experiment," Chinese Journal of Rock Mechanics and Engineering, vol. 32, no. 4, pp. 787-799, 2013. 
[13] H. Ji, H. Wang, S. Cao, Z. Hou, and Y. Jin, "Experimental research on frequency characteristics of acoustic emission signals under uniaxial compression of granite," Chinese Journal of Rock Mechanics and Engineering, vol. 31, no. 1, pp. 2900-2905, 2012.

[14] H. Ji and X. Lu, "Characteristics of acoustic emission and rock fracture precursors of granite under conventional triaxial," Chinese Journal of Rock Mechanics and Engineering, vol. 34, no. 4, pp. 694-702, 2015.

[15] J. Miao, M. He, D. Li, F. Zeng, and X. Zhang, "Acoustic emission characteristics of granite under strain rockburst test and its micro-fracture mechanism," Yanshilixue Yu Gongcheng Xuebao/Chinese Journal of Rock Mechanics and Engineering, vol. 28, no. 8, pp. 1593-1603, 2009.

[16] C. Gong, C.-H. Li, and K. Zhao, "Acoustic emission characteristics during short-time creep process of red sandstone under different stress level," Dongbei Daxue Xuebao, vol. 36, no. 9, pp. 1347-1352, 2015.

[17] C. P. Lu, L. M. Dou, H. Liu, H. S. Liu, B. Liu, and B. B. Du, "Case study on microseismic effect of coal and gas outburst process," International Journal of Rock Mechanics and Mining Sciences, vol. 53, pp. 101-110, 2012.

[18] X. Xu, L. Dou, C. Lu, and Y. Zhang, "Frequency spectrum analysis on micro-seismic signal of rock bursts induced by dynamic disturbance," Mining Science and Technology, vol. 20, no. 5, pp. 682-685, 2010.

[19] Y. Zhang, P. Liang, L. Sun, and X. Liu, "Frequency characteristics of acoustic emission signal during rupture process of siltstone based on HHT,' Caikuang yu Anquan Gongcheng Xuebao/Journal of Mining and Safety Engineering, vol. 33, no. 1, pp. 179-184, 2016.

[20] L. Dong, D. Sun, X. Li, and K. Du, "Theoretical and Experimental Studies of Localization Methodology for AE and Microseismic Sources Without Pre-Measured Wave Velocity in Mines," IEEE Access, vol. 5, pp. 16818-16828, 2017.

[21] L. Dong, W. Shu, X. Li, G. Han, and W. Zou, “Three Dimensional Comprehensive Analytical Solutions for Locating Sources of Sensor Networks in Unknown Velocity Mining System," IEEE Access, vol. 5, pp. 11337-11351, 2017.

[22] Z. W. Zeng, J. Ma, L. Q. Liu, and T. C. Liu, "AE b-value dynamic features of AE during rock mass fracturing and their significances," Journal of Seismology, vol. 17, pp. 7-11, 1995.

[23] P. M. Benson, S. Vinciguerra, P. G. Meredith, and R. P. Young, "Laboratory simulation of volcano seismicity," Science, vol. 322, no. 5899, pp. 249-252, 2008.

[24] L. Burlini, S. Vinciguerra, G. Di Toro, G. De Natale, P. Meredith, and J.-P. Burg, "Seismicity preceding volcanic eruptions: New experimental insights," Geology, vol. 35, no. 2, pp. 183-186, 2007.

[25] S. Q. Zhao, "Frequency spectra characteristics of acoustic emission signals during fracturing of rock specimen and a discussion of earthquake prediction," Advances in Science of China-Earth Science, pp. 19-34, 1986.

[26] C. H. Sondergeld and L. H. Estey, "Acoustic emission study of microfracturing during the cyclic loading of Westerly granite.," Journal of Geophysical Research: Atmospheres, vol. 86, no. 4, pp. 2915-2924, 1981.

[27] D. G. Aggelis, "Classification of cracking mode in concrete by acoustic emission parameters," Mechanics Research Communications, vol. 38, no. 3, pp. 153-157, 2011.

[28] N. Takai and Y. Mifune, "Digital watermarking by a holographic technique," Applied Optics, vol. 41, no. 5, pp. 865-873, 2002.
[29] Q. L. Li, T. Yu, and B. L. He, Temporal and Spatial Scanning for the Relation of Earthquake Frequency-Magnitude, Seismological Press, Beijing, China, 1979.

[30] X. Yin, S. Li, H. Tang, and J. Pei, "Study on quiet period and its fractal characteristics of rock failure acoustic emission," Yanshilixue Yu Gongcheng Xuebao/Chinese Journal of Rock Mechanics and Engineering, vol. 28, no. 2, pp. 3383-3390, 2009.

[31] X. Zhao, C. Tang, Y. Li, R. Yuan, and J. Zhang, "Study on AE activity characteristics under uniaxial compression loading," Yanshilixue Yu Gongcheng Xuebao/Chinese Journal of Rock Mechanics and Engineering, vol. 25, no. 2, pp. 3673-3678, 2006.

[32] S. Y. Li, T. M. He, and X. C. Yin, Introduction to rock fracture mechanics, University of Science and Technology of China, Hefei, China, 2010.

[33] X. C. Yin, Solid mechanics, Seismological Press, Beijing, China, 1985.

[34] Z. W. Zeng, J. Ma, X. Q. Wu, and A. F. Wang, "Characteristics and implications of acoustic emission energy in the process of deformation and failure of single-joint rockmass," Seismology \& Geology, vol. 16, no. 1, pp. 71-77, 1994.

[35] J. L. Miao, Experimental analysis of the energy characteristics of rockburst, China University of Mining and Technology, Beijing, China, 2009.

[36] Z. J. Wan and C. L. Zhou, "Study on the excitation model of rock acoustic emission source mechanism," Journal of Mining \& Safety Engineering, no. 4, pp. 67-69, 1998.

[37] S. D. Santis and A. K. Tomor, "Laboratory and field studies on the use of acoustic emission for masonry bridges," NDT \& E International, vol. 55, pp. 64-74, 2013.

[38] Y. H. Zou, Study of AE signal propagation mechanism of rock and coal, Shandong University of Science and Technology, Qingdao, China, 2007.

[39] M. F. Cai, M. C. He, and D. Y. Liu, Rock Mechanics and Rock Engineering, Science Press, Beijing, China, 2013.

[40] M. Ohnaka and K. Mogi, "Frequency characteristics of acoustic emission in rocks under uniaxial compression and its relation to the fracturing process to failure.," Journal of Geophysical Research: Atmospheres, vol. 87, no. 5, pp. 3873-3884, 1982.

[41] M. Ohnaka and K. Mogi, "Frequency Dependence of Acoustic Emission Activity in Rocks under Incremental Uniaxial Compression," Bull Earthquake Research Institute, vol. 56, pp. 67-89, 1981. 


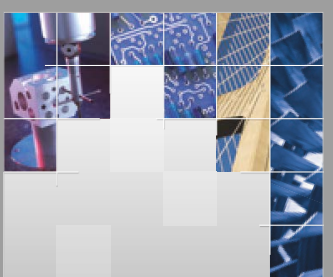

\section{Enfincering}
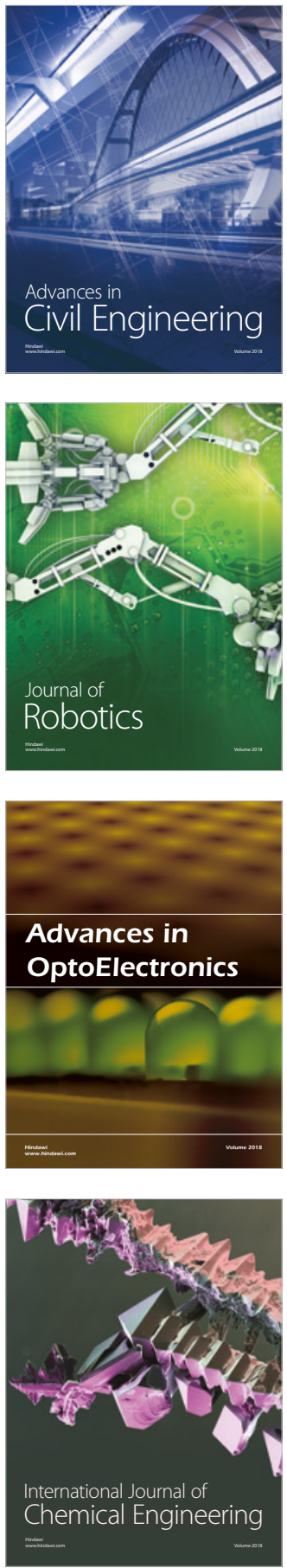

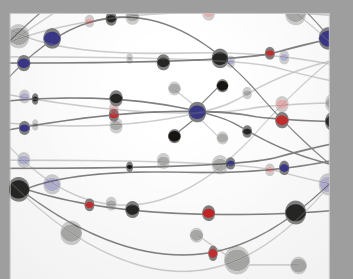

\section{Rotating \\ Machinery}

The Scientific World Journal

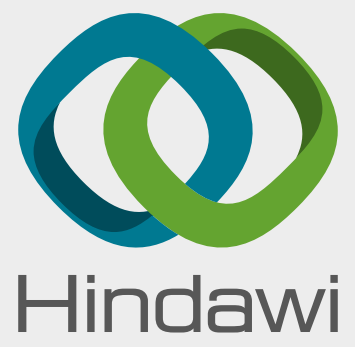

Submit your manuscripts at

www.hindawi.com
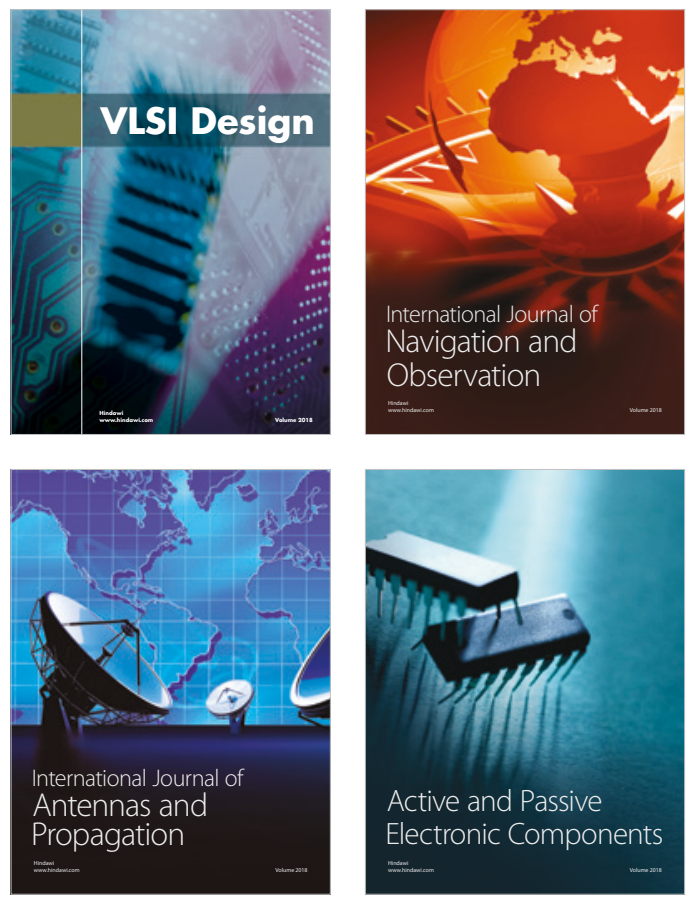
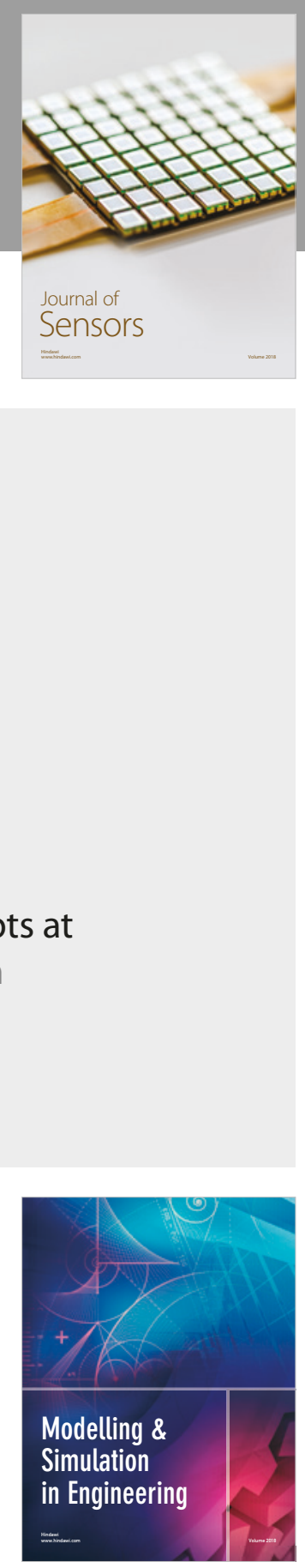

\section{Advances \\ Multimedia}
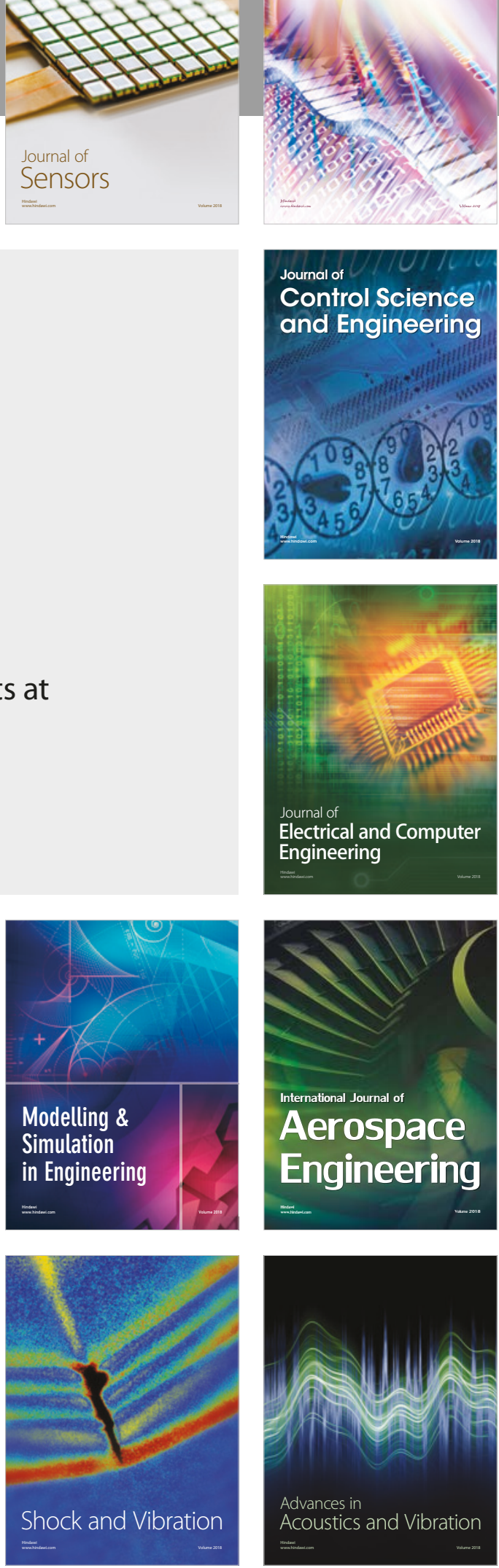